\title{
MUSINGS
}

\section{No small matter: qualitatively distinct challenges of pediatric genomic studies}

\author{
Isaac S Kohane*
}

\section{The importance of pediatric genomics studies}

There are a number of compelling reasons why pediatric populations should be preferentially studied to understand the genomic basis of disease. First, many forms of adult disease have genetic etiologies that were discovered in pediatric studies (for example, cardiomyopathies, several causes of infertility and colorectal cancer) where the more penetrant or homozygous mutations, clinically presenting in early childhood, made the genetic pathogenesis much clearer than in adult disease. Second, being able to detect the genetic antecedents of adult disease best allows us to study how the disease develops on the way to adulthood and, just as importantly, gives the longest possible lead time for the implementation of the most cost-effective primary prevention (for example, use of angiotensin converting enzyme inhibitors to reduce the cardiac manifestations of Marfan syndrome if given in childhood). Third, the individual variation due to environmental exposures, whether it be diet, drugs or other habits, is by definition relatively small compared with that of adults who have had an order of magnitude more exposure to the environment. Consequently, the case could be made that a greater fraction of pathophysiological variability in children can be attributable to inherited traits (although one could make several opposing arguments, including the increased susceptibility of children to certain environmental insults). Last, and most importantly, children constitute a population with distinct physiology and disease risks and there is no substitute for pediatric genomics studies in order to better understand and ultimately manage or treat these risks and diseases. So why is it that pediatric genomic studies form only a small fraction of the entire collection of genomic studies formed to date?

\section{Challenges in pediatric genomics research}

There is more here than just the usual underfunding of pediatric projects relative to adult projects, although this

*Correspondence: Isaac_Kohane@hms.harvard.edu

Children's Hospital Informatics Program and Harvard Medical School Center for Biomedical Informatics, Boston, MA 02115, USA certainly may be an important factor [1]. In many ways the barriers mirror some of those that cause underrepresentation of historically under-represented and underserved minorities in genetic studies as outlined by Francis Collins and colleagues [2]. One important consideration is that it is just much harder to perform genetic studies with children. To start with, there is the matter of consent and assent. Children are not children forever and therefore the parental consent most likely has to be eventually replaced by childhood assent and then full consent as they reach maturity [3]. This already imposes significantly more in terms of overheads for consent management than those incurred by adult prospective studies.

Then there is the challenge of obtaining the biological sample. In the judgment of many parents, most children, and a few institutional review boards, the pain and small risks of venipuncture for blood samples outweigh potential benefits, particularly for healthy children. The alternative (for example, obtaining saliva as a source of DNA) often results in suboptimal genomic analyses due to difficulties in obtaining an adequate quantity of sample in young children.

In addition, most pediatric care is delivered in small practices, and much of this care and ancillary measurements are not documented in the electronic health records that are mostly found in larger healthcare systems. This makes identification of cases and controls largely an expensive and manual operation. Moreover, the transition to adulthood almost always entails a change in healthcare provider and healthcare delivery system and therefore a discontinuity in record keeping (electronic and/or paper). This results in loss of follow-up information that is essential for genomic studies that address long-term outcomes.

Perhaps most challenging is that there is not one population of children but many populations with truly distinct physiologies: neonates have dramatically different 'normal' ranges (for example, for ionic and endocrine analytes) and physiologies (for example, relative dependence on glucose as a primary metabolic fuel) compared with even a 1 month old. Early childhood, puberty and adolescence also constitute such different physiological 
states as to cause the population differences usually considered in genomic studies to pale by comparison.

\section{Overcoming the challenges}

These barriers are not insurmountable and several steps can be taken now to address them. First, any pediatric research institution should implement a routine consent tracking system designed for children growing up through adulthood, and which allows for all the complexities of maturation of autonomy and changes in guardianship and family structure. Second, the chemistries for sample preparation for 'next generation' sequencing can and likely will be optimized to yield high-quality genomic data from much smaller samples of cellular material in saliva or easily brushed off skin. Perhaps even more so than for adult studies, every opportunity to study and measure children as they progress through the various states of development has to be fully leveraged, while maximizing the autonomy and privacy of the child and his/her family, by accelerating the adoption of pediatric electronic health records and the infrastructure required [4]. Finally, the transition of record keeping from pediatric to adult care must be addressed whether by mandate [5] or by implementation of national or regionally integrated health records or even by patient-driven solutions such as personally controlled health records [6]. With regard to the latter, pediatricians have long implemented the paper-based equivalent of a personally controlled record (that is, a paper log of all immunizations and blood tests, and occasionally major anthropometric measures) and placed them in the possession of parents. However, these frequently misplaced paper records are increasingly being replaced by electronic equivalents despite ongoing concerns regarding electronic access and health literacy. With the concurrent growth in large institutional and national biobanks, there is an opportunity to merge integrated health records with the biomaterials in these biobanks [7] and thereby accelerate pediatric genomic studies. The same institutions and countries are also well positioned to set the standard internationally for both the information technology practices and the consenting and consent-tracking practices that are required for pediatric studies. Such an international collaborative effort will be necessary to achieve sufficient sample sizes of at least 100,000 for important pediatric quantitative traits.
Finally, the strategies outlined above depend on a productive professional relationship between primary care pediatricians (who will be providing the bulk of clinical characterization) and genome-scale researchers. Successful incentive models whether financial or through academic attribution and advancement have been elusive in the face of ongoing pediatrician concern regarding extra work, lack of resources, lack of rewards and recognition, difficulty in providing informed consent, and fear of losing patients [8]. My own intuition is that a commitment on the part of researchers to interpret novel and pertinent genetic findings in a manner that can easily be shared with the patients to which the findings apply will create an added incentive [9] that will augment the currently tenuous bond between pediatrician, researcher and patient that is essential to pediatric genomic research.

\section{Competing interests}

The author declares that he has no competing interests.

\section{Acknowledgements}

The author is indebted to Drs Ingrid Holm and David Margulies for insightful comments and helpful edits.

Published: 30 September 2011

\section{References}

1. Hay WW Jr: American Pediatric Society presidential address 2008: research in early life - benefit and promise. Pediatr Res 2009, 65:117-122.

2. Collins FS, Green ED, Guttmacher AE, Guyer MS; US National Human Genome Research Institute: A vision for the future of genomics research. Nature 2003, 422:835-847.

3. Bourgeois FC, Taylor PL, Emans SJ, Nigrin DJ, Mandl KD: Whose personal control? Creating private, personally controlled health records for pediatric and adolescent patients. J Am Med Inform Assoc 2008, 15:737-743.

4. Kohane IS, Mandl KD, Taylor PL, Holm IA, Nigrin DJ, Kunkel LM: Medicine. Reestablishing the researcher-patient compact. Science 2007, 316:836-837.

5. Blumenthal D, Tavenner M: The 'meaningful use' regulation for electronic health records. N Engl J Med 2010, 363:501-504.

6. Mandl KD, Kohane IS: Tectonic shifts in the health information economy. NEngl J Med 2008, 358:1732-1737.

7. Kohane IS: Using electronic health records to drive discovery in disease genomics. Nat Rev Genet 2011, 12:417-428.

8. Caldwell PH, Murphy SB, Butow PN, Craig JC: Clinical trials in children. Lancet 2004, 364:803-811

9. Kaufman D, Murphy J, Scott J, Hudson K: Subjects matter: a survey of public opinions about a large genetic cohort study. Genet Med 2008, 10:831-839.

doi:10.1186/gm 278

Cite this article as: Kohane IS: No small matter: qualitatively distinct challenges of pediatric genomic studies. Genome Medicine 2011, 3:62 